\title{
Evidence of Andreev bound states as a hallmark of the FFLO phase in $\kappa$-(BEDT-TTF $)_{2} \mathrm{Cu}(\mathrm{NCS})_{2}$
}

\author{
H. Mayaffre ${ }^{1}$, S. Krämer ${ }^{1}$, M. Horvatić ${ }^{1}$, C. Berthier ${ }^{1}$, K. Miyagawa ${ }^{2}$, K. Kanoda ${ }^{2}$ and V. F. Mitrović ${ }^{3 \star}$
}

Superconductivity is a quantum phenomenon arising, in its simplest form, from the pairing of fermions with opposite spin into a state with zero net momentum. Whether superconductivity can occur in fermionic systems with an unequal number of two species distinguished by spin or flavour presents an important open question in condensed-matter physics or quantum chromodynamics'. In condensed matter the imbalance between spin-up and spin-down electrons that form the Cooper pairs is induced by the magnetic field. Such an imbalanced system can lead to exotic superconductivity in which pairs acquire finite momentum ${ }^{2,3}$. This momentum leads to a spatially inhomogeneous state consisting of periodically alternating 'normal' and 'superconducting' regions. Here, we establish that the hallmark of this state is the appearance of spatially localized and spin-polarized quasiparticles forming the so-called Andreev bound states (ABS). These are detected through our nuclear magnetic resonance (NMR) measurements.

The Fulde-Ferrell-Larkin-Ovchinnikov (FFLO) phase is expected to occur in the vicinity of the upper critical magnetic field $\left(H_{\mathrm{c} 2}\right)$ when Pauli pair breaking dominates over orbital (vortex) effects $^{2-5}$. Pauli pair breaking prevails in fields that exceed the Pauli limit $\left(H_{\mathrm{p}}\right)$ for which the Zeeman energy is strong enough to break the Cooper pair by flipping one spin of the singlet. Intense efforts have been invested to search for indisputable evidence for the existence of the FFLO states. Examples include a theoretical proposal for detecting modulated superfluid phases in optical lattices ${ }^{6}$; tunnelling in superconducting (SC) films ${ }^{7}$; mapping of the

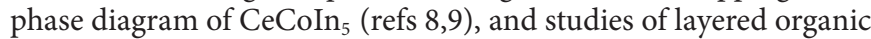
superconductors ${ }^{10-13}$. However, clear microscopic evidence for an FFLO phase is still missing. In the FFLO state, in the vicinity of the transition from the SC to FFLO state, nodes in the order parameter form the domain walls, where the superconducting phase changes by $\pi$. This phase twist leads to a local modification of the electronic density of states (DOS) and the creation of new topological ABS (ref. 14), the observation of which we report here.

Besides $\mathrm{CeCoIn}_{5}$, where a putative FFLO state coexists with long-range magnetism, the organic compound, $\kappa$-(BEDT$\mathrm{TTF})_{2} \mathrm{Cu}(\mathrm{NCS})_{2}$ (hereafter referred to as $\left.\kappa-(\mathrm{ET})_{2} \mathrm{X}\right)$ exhibits the clearest thermodynamic evidence for the existence of a narrow intermediate SC phase ${ }^{11}$. Because this SC phase is stabilized in magnetic fields $(H)$ that exceed the Pauli limit, $H_{\mathrm{p}} \approx 20.7 \mathrm{~T}$ (ref. 15), as illustrated in Fig. 1, it has been identified as an FFLO phase. Recent measurements of NMR spectra gave evidence that the phase transition within the SC state is Zeeman-driven ${ }^{16}$, but failed to provide a clear hallmark of the FFLO state. Our main discovery is that the NMR spin-lattice relaxation rate $\left(T_{1}^{-1}\right)$ becomes

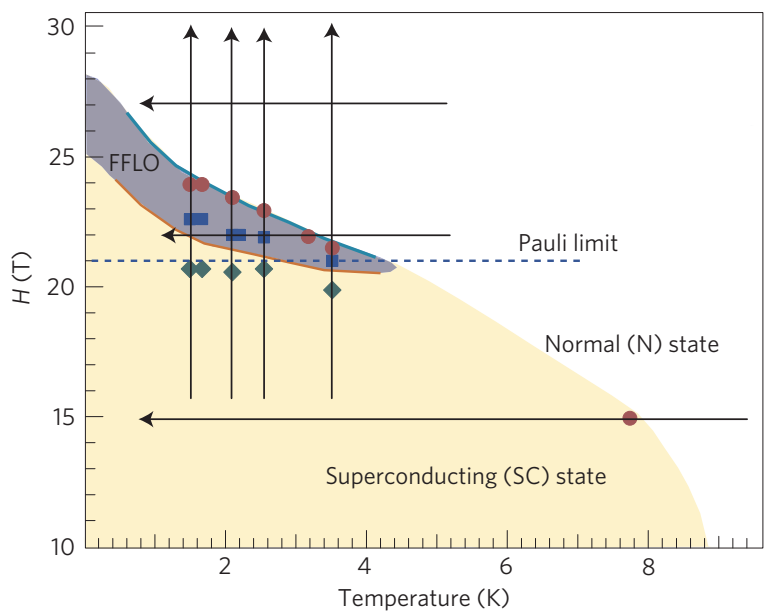

Figure $1 \mid(H, T)$ phase diagram of $\kappa$-(BEDT-TTF $)_{2} \mathrm{Cu}(\mathrm{NCS})_{2}$. Curves and colour shaded areas sketch the phase diagram based on magnetic torque measurements ${ }^{12}$ for fields parallel to the conducting planes. Circles denote $T_{\mathrm{c}}$, the transition temperature from the normal to the SC state, determined from our NMR data, as explained in the text, whereas squares mark the peak in the NMR rate. Diamonds denote the onset $H$ above which $\left(T_{1} T\right)^{-1}$ exceeds the value extrapolated from the low-field SC state (Supplementary Fig. 6 and Supplementary Information) below $H_{p} \approx 20.7 T$ (ref. 15). Arrows indicate the field $(H)$ and temperature $(T)$ scans covered by NMR relaxation rate measurements.

significantly enhanced, as compared to its normal-state value, in the SC state for fields exceeding $H_{\mathrm{p}}$. We deduce that the enhancement stems from the ABS of polarized quasiparticles spatially localized in the nodes of the order parameter in an FFLO state. Furthermore, we reveal that these topological ABS are profoundly different from the sub-gap states found in vortex cores, as they are shifted in energy away from the Fermi level by an amount controlled by the magnetic field.

We first examine the NMR spectral lineshapes in different regimes at $22 \mathrm{~T}$, shown in the inset to Fig. 2, to demonstrate the sensitivity of our measurements to different superconducting phases in this compound. These ${ }^{13} \mathrm{C}$ NMR spectra reflect the distribution of the hyperfine fields and are thus a sensitive probe of the electronic spin polarization ${ }^{16-18}$. In the normal state at $10.9 \mathrm{~K}$ the spectrum is relatively broad and exhibits multiple peaks corresponding to inequivalent ${ }^{13} \mathrm{C}$ spin-labelled sites ${ }^{17,19,20}$. At $1.4 \mathrm{~K}$, deep in the superconducting state, the spectrum is significantly narrower than in the normal state. This narrowing is due to the decrease of the

\footnotetext{
${ }^{1}$ Laboratoire National des Champs Magnétiques Intenses, LNCMI - CNRS (UPR 3228), UJF, UPS and INSA, BP 166, 38042 Grenoble Cedex 9 , France. ${ }^{2}$ Department of Applied Physics, University of Tokyo, Bunkyo-ku, Tokyo 113-8656, Japan. ${ }^{3}$ Department of Physics, Brown University, Providence, Rhode Island 02912, USA. *e-mail: vemi@brown.edu
} 


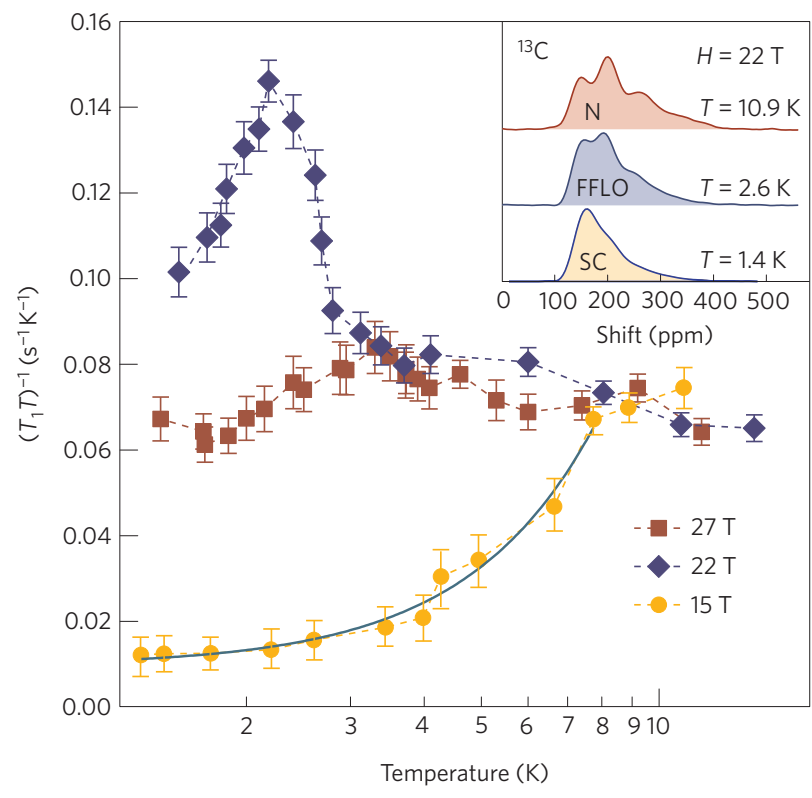

Figure 2 | NMR relaxation rate in the normal and superconducting states. Temperature dependence of ${ }^{13} \mathrm{C}$ NMR $\left(T_{1} T\right)^{-1}$ at fields of 15, 22 and $27 \mathrm{~T}$, applied in the conducting planes (symbols). Solid line denotes the quadratic temperature dependence characteristic for superconductors with a gap having a line of nodes, such as for a $d$-wave symmetry. The dashed lines are guides to the eye. The parts of the phase diagram explored are shown by horizontal arrows in Fig. 1. Error bars reflect the scattering of measured $\left(T_{1} T\right)^{-1}$ values and the standard error of the mean in fitting the recovery curve. Inset: ${ }^{13} \mathrm{C}$ NMR spectra at $22 \mathrm{~T}$ field applied parallel to the conducting planes in the superconducting $(T=1.4 \mathrm{~K}), \mathrm{FFLO}(T=2.6 \mathrm{~K})$, and normal state $(T=10.9 \mathrm{~K})$. Multiple peaks evident in the normal-state spectrum arise from the eight distinct crystallographic sites of ${ }^{13} \mathrm{C}$ (Methods).

average electronic spin polarization in the superconducting state with singlet pairing, which in turn reduces the splitting between the lines corresponding to distinct carbon sites. At $2.6 \mathrm{~K}$, in the putative FFLO state, the spectrum is narrower than in the normal state yet wider than deep in the superconducting state. This indicates that the sample is indeed in the SC state at this temperature and that the DOS at the Fermi energy $\left(E_{\mathrm{F}}\right)$ is suppressed below $T_{\mathrm{c}}$. Thus, whatever the nature of the high-field superconducting state is, its DOS at $E_{\mathrm{F}}$ (averaged over the sample volume) is suppressed as compared to the normal state.

The temperature dependence of $\left(T_{1} T\right)^{-1}$ measured at various magnetic fields, applied in the conducting planes, is plotted in Fig. 2. In the normal state $\left(T_{1} T\right)^{-1}$ is constant, indicating that the DOS in the vicinity of $E_{\mathrm{F}}$ is constant (Supplementary Information) $)^{21}$. At $27 \mathrm{~T},\left(T_{1} T\right)^{-1}$ remains nearly constant within the error bars, which implies that the sample remains in the normal state down to the lowest temperature $(1.3 \mathrm{~K})$ that we investigated. At $15 \mathrm{~T}$, the rate decreases below $T_{\mathrm{c}} \approx 8 \mathrm{~K}$, evidencing formation of the singlet SC state. At an intermediate field of $22 \mathrm{~T}$, the sample remains in the normal state down to $T_{\mathrm{c}} \approx 3 \mathrm{~K}$. Below $T_{\mathrm{c}},\left(T_{1} T\right)^{-1}$ increases sharply to nearly twice the normal-state value, reaching a maximum in the vicinity of $2 \mathrm{~K}$.

The magnetic field dependence of $\left(T_{1} T\right)^{-1}$ at various temperatures is plotted in Fig. 3. In the normal state above $25 \mathrm{~T},\left(T_{1} T\right)^{-1}$ is independent of both field and temperature. Below $21 \mathrm{~T},\left(T_{1} T\right)^{-1}$ is suppressed under its normal-state value owing to the formation of singlet superconductivity. The enhancement of $\left(T_{1} T\right)^{-1}$ is observed for the intermediate field values from 21 to $24 \mathrm{~T}$. The observed enhancement of $\left(T_{1} T\right)^{-1}$ is stunning because it appears in the SC state only in fields exceeding $H_{\mathrm{p}}$ and where spectral measurements

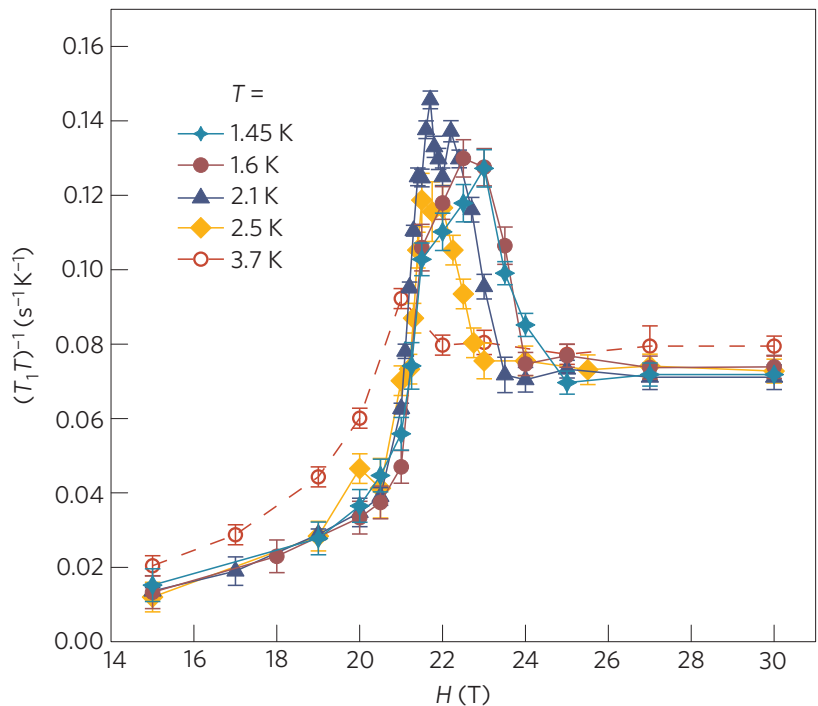

Figure 3 | Enhancement of the NMR relaxation rate in the FFLO state. Magnetic field dependence of ${ }^{13} \mathrm{C} \mathrm{NMR}\left(T_{1} T\right)^{-1}$ at various temperatures. The parts of the phase diagram explored are shown by vertical arrows in Fig. 1. Error bars reflect the scattering of measured $\left(T_{1} T\right)^{-1}$ values and the standard error of the mean in fitting the recovery curve.

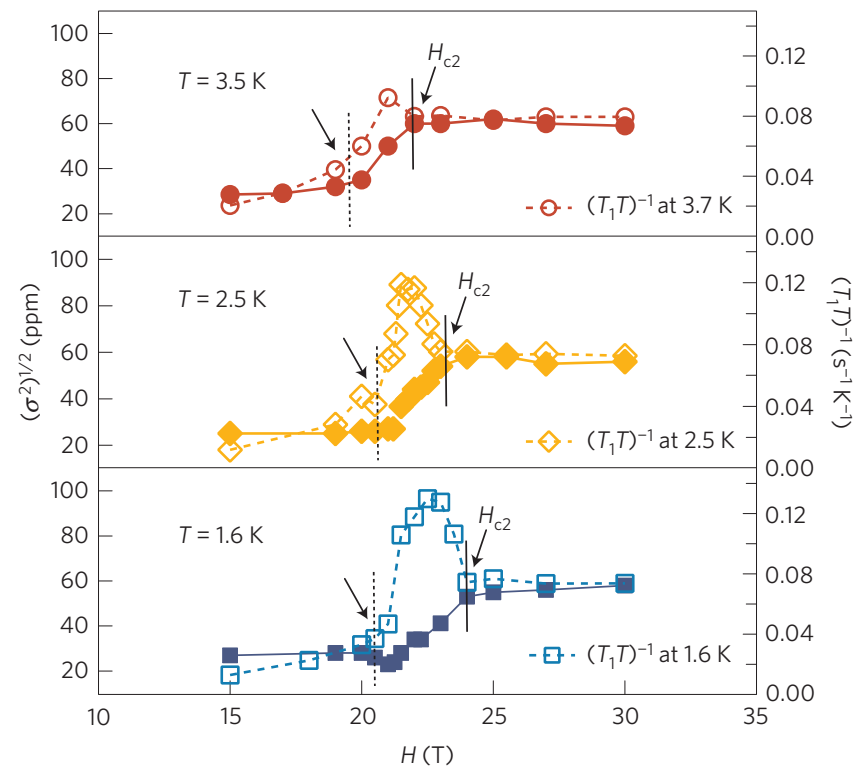

Figure 4 | Field dependence of the electronic spin polarization and NMR relaxation rate at low temperatures. Square root of the second moment, measuring electronic spin polarization, of the ${ }^{13} \mathrm{C}$ NMR spectra (filled symbols, left scale) as a function of magnetic field applied in the conducting planes at different temperatures. Typical error bars are on the order of a few per cent and not shown for clarity. The corresponding field dependence of $\left(T_{1} T\right)^{-1}$ (open symbols, right scale) is shown for comparison. Vertical dashed lines identify a possible transition from the FFLO to the homogeneous SC state. Vertical solid lines denote a transition from the normal to the FFLO state. Other lines are guides to the eye.

indicate suppression of the DOS at the $E_{\mathrm{F}}$, as shown in the inset to Fig. 2 and Fig. 4.

An important question is whether the observed enhancement of $\left(T_{1} T\right)^{-1}$ in a SC state can be explained by more 'standard' mechanisms amplifying the NMR relaxation in a SC state, for example, due to vortices. In the following we show that such 


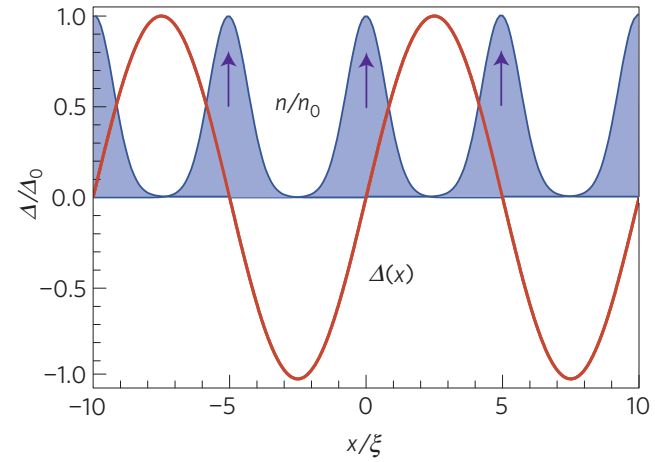

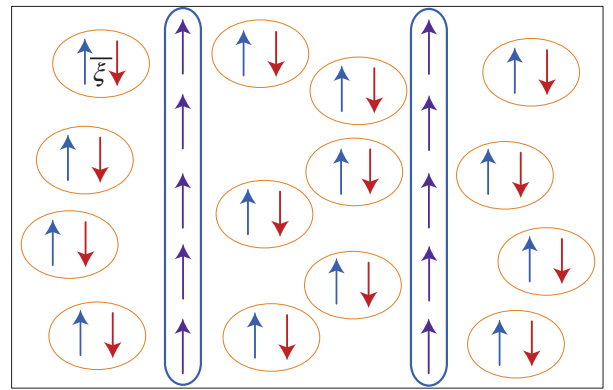

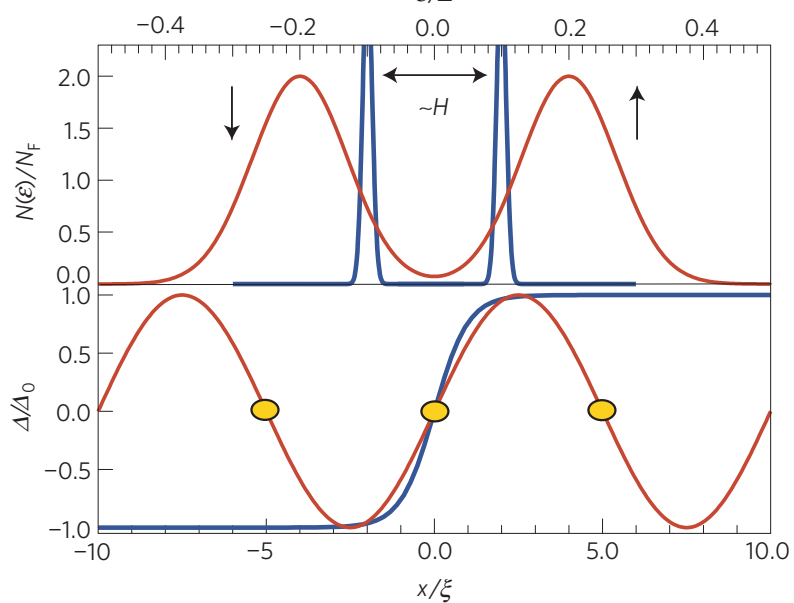

d

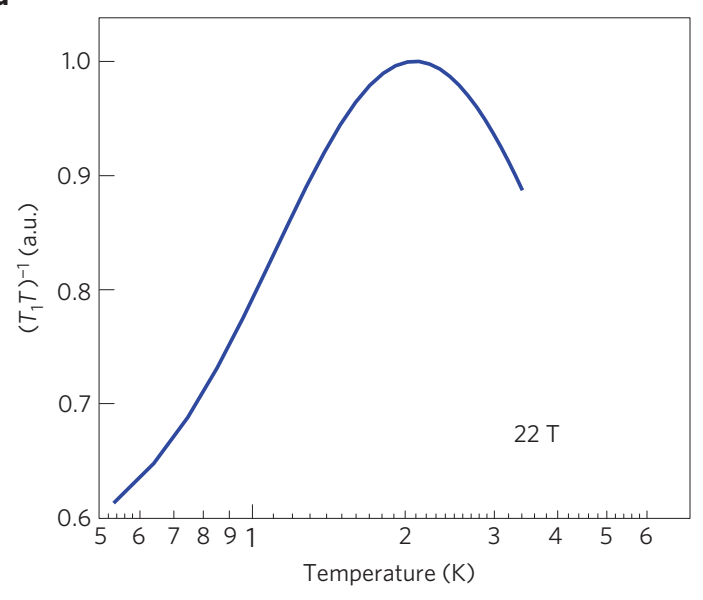

Figure $\mathbf{5}$ | Schematic of the properties of the modulated superconductivity. $\mathbf{a}$, Sketch of the spatial profile of the order parameter and density of the localized polarized quasiparticles in length units normalized by the superconducting coherence length $(\xi)$. $\mathbf{b}$, Schematic of the spatial structure of the modulated superconducting states with excess polarized quasiparticles in the nodes of the order parameter. c, Bottom panel: Sketch of the spatial profile of the order parameter for two different magnetic fields. In the vicinity of the transition from the SC to FFLO state the nodes in the order parameter form the domain walls ${ }^{14}$, as illustrated by the blue solid line. With increasing magnetic field the period of the order parameter decreases, as shown by red lines. Yellow circles denote nodal regions. Top panel: Sketch of the local DOS (ABS formed by quasiparticle resonances) at nodes of the order parameter normalized to its normal-state value. Sharp DOS peaks (blue) are expected in magnetic fields where nodes in the order parameter form the domain walls ${ }^{14}$. As the applied field increases, ABS are broadened and further shifted from zero energy, as depicted by red lines. The vertical arrows denote the spin orientation. The energy difference between spin-up and -down states is proportional to the applied magnetic field. $\mathbf{d}$, Calculated temperature dependence (in arbitrary units (a.u.)) of the $\left(T_{1} T\right)^{-1}$ arising from the Andreev bound states formed at the nodes of the order parameter as sketched in part $\mathbf{c}$. The result is only qualitatively correct, as states are phenomenologically modelled by Gaussians shifted away from the Fermi level by an amount proportional to the applied field ${ }^{14}$ and the temperature dependence of the gap is neglected.

mechanisms can be readily discarded (see also Supplementary Information). Vortices can provide two relaxation channels: one due to quasiparticles in vortex cores and the other from field fluctuations induced by vortex motion. Our measurements have been carried out for the applied field being precisely parallel to the conducting planes, as identified by the minimum in $T_{1}^{-1}$ (Methods and Supplementary Information). This minimum implies that only Josephson-like vortices exist; these 'coreless' vortices ${ }^{22}$ contain no quasiparticles and thus do not contribute to $T_{1}^{-1}$ (refs 18,23). As a matter of fact, it was shown that in this 'coreless' vortex state $T_{1}^{-1}$ contains only a small background contribution ${ }^{18,23}$, which corresponds to that measured in our experiment at $15 \mathrm{~T}$ at lowest temperatures deep in the superconducting state, and is nearly ten times smaller than $T_{1}^{-1}$ measured in $22 \mathrm{~T}$. As for the vortex motion, the Josephson vortices are formed in the insulating layers and, strictly speaking, magnetic flux lines do not penetrate the SC layers ${ }^{24}$. Thus, even though these vortices are only weakly pinned ${ }^{25}$, their motion should not significantly contribute to the ${ }^{13} \mathrm{C}_{1}^{-1}$ rate, dominated by the hyperfine coupling to the electronic degrees of freedom of the SC planes ${ }^{24}$ (Supplementary Information).
In the absence of magnetic correlation, the NMR relaxation rate is given by

$$
\frac{1}{T_{1} T} \propto \frac{1}{T} \int \mathrm{d} \varepsilon N_{\uparrow}(\varepsilon) N_{\downarrow}(\varepsilon) f(\varepsilon)[1-f(\varepsilon)]
$$

where $N_{\downarrow}$ and $N_{\uparrow}$ denote the densities of up- and down-spin states and $f(\varepsilon)$ is the Fermi occupation function. That is, $\left(T_{1} T\right)^{-1}$ is proportional to the square of the DOS integrated over energy $\varepsilon$ in a range of the order of $k_{\mathrm{B}} T$ around $E_{F}$ selected by the Fermi function. This implies that in a SC state with the nodes in the gap, at low temperature only the DOS in the regions around the nodes contributes to the relaxation rate. For energies less than half of the gap magnitude, the DOS near the nodes depends linearly on quasiparticle excitation energy, leading to well-known $T^{2}$ dependence of the $\left(T_{1} T\right)^{-1}$, illustrated in Fig. 2. This decrease of $\left(T_{1} T\right)^{-1}$ with decreasing temperature in the SC state is a direct consequence of the lack of low-energy features in the DOS. A more complex temperature dependence of the relaxation rate, such as that observed in fields above $H_{\mathrm{p}} \approx 21 \mathrm{~T}$, can be generated by a peak-like 
structure in the DOS at low energies around $E_{\mathrm{F}}$. The question remains as to what gives rise to such DOS features (that is bound states). The bound states can form only in regions where the SC order parameter is suppressed. As the presence of vortex cores in our experiment was excluded, the ABS formed near the zeros of the FFLO order parameter ${ }^{14,26-29}$ provide a natural explanation. In fact, a sharp peak-like structure around $E_{\mathrm{F}}$ is predicted in the FFLO state in the vicinity of the transition from the SC to the FFLO state, where nodes in the order parameter form the domain walls ${ }^{14}$, as illustrated in Fig. 5c. The qualitative temperature dependence of $\left(T_{1} T\right)^{-1}$ arising from such sub-gap bound states is shown in Fig. $5 \mathrm{~d}$. Evidently, it is in sharp contrast to previously discussed standard behaviour for a $d$-wave SC state at lower fields.

To emphasize our main finding that the observed enhancement of $\left(T_{1} T\right)^{-1}$ in the high-field superconducting state indicates the presence of the ABS formed near the zeros of the FFLO order parameter, in Fig. 4 we plot the square root of the second moment of the lineshape, as a quantitative measure of the width of inhomogeneous spectra, together with the $\left(T_{1} T\right)^{-1}$ data. It is evident that the onset of the $\left(T_{1} T\right)^{-1}$ enhancement is concurrent with the spectral line narrowing generated by the decreasing electronic spin polarization (and, thus, the DOS at $E_{\mathrm{F}}$ ) in the SC state. That is, both the DOS at $E_{\mathrm{F}}$ and the average spin polarization are lower than in the normal state. Thus, the $\left(T_{1} T\right)^{-1}$ enhancement over the normal-state value is to be assigned exclusively to quasiparticle bound states located away from $E_{\mathrm{F}}$. Such bound states shifted away from $E_{\mathrm{F}}$, forming in applied fields exceeding $H_{\mathrm{p}}$, are the hallmark of an FFLO state ${ }^{14}$. Because these states are localized in the real space in the nodal region, they occupy a small fraction-of the order of several per cent of the total sample volume (as sketched in Fig. 5b); even if they were to produce a finite DOS at $E_{\mathrm{F}}$, they would not contribute to any significant broadening or shift of the NMR spectra, which reflect the average over the entire sample. However, such a localized DOS can affect the global NMR rate as a result of either nuclear spin diffusion, 'transferring' the effect from nuclei spatially localized in the nodes to those far outside as well, or motion of the nodal planes in which bound states of polarized quasiparticles are localized (Supplementary Information).

The increasing $\left(T_{1} T\right)^{-1}$ with decreasing temperature is a direct consequence of the appearance of the sharp (compared to $k_{\mathrm{B}} T$ ) bound states located away from $E_{\mathrm{F}}$. The energy position and width of these bound states is set by the applied field, as described in detail in Methods. At a given field, the only effect of temperature on $\left(T_{1} T\right)^{-1}$ is to vary the range $\varepsilon \simeq k_{\mathrm{B}} T$ around $E_{\mathrm{F}}$ over which the square of the DOS is averaged in equation (1). Thus, on lowering temperature in the FFLO state at fixed field, $\left(T_{1} T\right)^{-1}$ first increases as the bound states are created just below $T_{c}$. For temperatures below which the bound state DOS energy exceeds $\varepsilon,\left(T_{1} T\right)^{-1}$ will decrease. This is in qualitative agreement with the observed temperature dependence of $\left(T_{1} T\right)^{-1}$ at $22 \mathrm{~T}$, in the FFLO state, plotted in Fig. 2 (Methods).

\section{Methods}

Sample \& NMR methods. Experiments were performed on high-quality $\kappa$-(ET) $)_{2} \mathrm{X}$ single crystals, grown by an electrolytic method ${ }^{30}$. The most suitable nucleus for our NMR study is ${ }^{13} \mathrm{C}$. As it has a low natural abundance, we used samples selectively enriched with ${ }^{13} \mathrm{C}$ on the site that is the most sensitive to electronic degrees of freedom. Such sites are located on the central C-C pair that bears the largest spin density in the molecule ${ }^{17}$. We used samples with $100 \%$ ${ }^{13} \mathrm{C}$-enriched pairs, giving rise to eight NMR inequivalent sites. The sample, placed inside the radio-frequency NMR coil, was oriented by an accurate mechanical goniometer. As the field must be applied strictly parallel to the conducting planes with a precision better than $1.4^{\circ}$ for the possible FFLO phase to be stabilized ${ }^{12}$, a sharp minimum in the $T_{1}^{-1}$ was used as sensitive in situ signature of the precise alignment of the field within the conduction planes ${ }^{23}$ (see also Supplementary Information). That is, when the field is exactly aligned along the conducting planes only Josephson-type vortices can form. In the core of such vortices quasiparticles are depleted, leading to a significant suppression of $T_{1}^{-1}$ (refs 18,23).
The measurements were done at the LNCMI in Grenoble, using a superconducting magnet for $H=15 \mathrm{~T}$ and a resistive magnet at higher fields. The temperature control was provided by a ${ }^{4} \mathrm{He}$ variable temperature insert. The NMR data were recorded using a state-of-the-art laboratory-made NMR spectrometer. $T_{1}^{-1}$ was measured by the saturation-recovery method: following the saturation of nuclear magnetization obtained by applying a train of $\pi / 2$ pulses equally spaced by a time $t \geq T_{2}$, the signal was detected after a variable delay time using a standard spin-echo sequence $(\pi / 2-\tau-\pi)$.

$T_{\mathrm{c}}$ determination. $T_{\mathrm{c}}$ was identified by examining the NMR spectral shapes and shifts, and the tuning resonance of the NMR tank circuit.

Field dependence of $\left(T_{1} T\right)^{-1}$. Compared to the temperature dependence of $\left(T_{1} T\right)^{-1}$, a quantitative explanation and comparison of the field dependence is more difficult as the effect of the field is twofold. In addition to shifting the bound states away from $E_{\mathrm{F}}$, the applied field controls the sharpness of these states (as depicted in Fig. 5c). That is, as the field increases and more nodal planes are introduced, bound states broaden in energy as a consequence of hybridization of the energy levels corresponding to the adjacent planes. As a result, the sharp bound state for a single domain wall (formed near the transition from the SC state) broadens and thus fills the low-energy region below the maximum gap. At a given temperature, it is the interplay of the broadening and the peak energy of the bound states, both controlled by the field, that determines the field value at which the peak in $\left(T_{1} T\right)^{-1}$ is observed.

\section{Received 7 October 2013; accepted 11 September 2014;} published online 26 October 2014

\section{References}

1. Casalbuoni, R. \& Nardulli, G. Inhomogeneous superconductivity in condensed matter and QCD. Rev. Mod. Phys. 76, 263-320 (2004).

2. Fulde, P. \& Ferrell, R. A. Superconductivity in a strong spin-exchange field. Phys. Rev. 135, A550-A564 (1964).

3. Larkin, A. I. \& Ovchinnikov, Y. N. Inhomogeneous state of superconductors Sov. Phys. JETP 20, 762-769 (1965).

4. Maki, K. Effect of Pauli paramagnetism on magnetic properties of high-field superconductors. Phys. Rev. 148, 362-369 (1966).

5. Gruenberg, L. \& Gunther, L. Fulde-Ferrell effects in Type-II superconductors. Phys. Rev. Lett. 16, 996-998 (1966).

6. Loh, Y. L. \& Trivedi, N. Detecting the elusive Larkin-Ovchinnikov modulated superfluid phases for imbalanced Fermi gases in optical lattices. Phys. Rev. Lett. 104, 165302 (2010).

7. Loh, Y. L., Trivedi, N., Xiong, Y. M. P. W., Adams, \& Catelani, G. Origin of excess low-energy states in a disordered superconductor in a Zeeman field. Phys. Rev. Lett. 107, 067003 (2011).

8. Bianchi, A., Movshovich, R., Capan, C., Pagliuso, P. G. \& Sarrao, J. L. Possible Fulde-Ferrell-Larkin-Ovchinnikov superconducting state in $\mathrm{CeCoIn}_{5}$. Phys. Rev. Lett. 91, 187004 (2003).

9. Koutroulakis, G. et al. Field evolution of coexisting superconducting and magnetic orders in CeCoIn ${ }_{5}$. Phys. Rev. Lett. 104, 087001 (2010).

10. Singleton, J. et al. Observation of the Fulde-Ferrell-Larkin-Ovchinnikov state in the quasi-two-dimensional organic superconductor $\kappa-(\mathrm{BEDT})_{2}(\mathrm{CuSCN})_{2}(\mathrm{BEDT}-\mathrm{TTF} \equiv$ bis(ethylene-dithio)tetrathiafulvalene). J. Phys. Condens. Matter 12, L641-L648 (2000).

11. Lortz, R. et al. Calorimetric evidence for a Fulde-Ferrell-Larkin-Ovchinnikov superconducting state in the layered organic superconductor $\kappa$-(BEDT-TTF $)_{2}(\mathrm{CuNCS})_{2}$. Phys. Rev. Lett. 99, 187002 (2007).

12. Bergk, B. et al. Magnetic torque evidence for the Fulde-Ferrell-Larkin-Ovchinnikov state in the layered organic superconductor $\kappa$-(BEDT-TTF) ${ }_{2}(\mathrm{CuNCS})_{2}$. Phys. Rev. B 83, 064506 (2011).

13. Uji, S. et al. Vortex dynamics and the Fulde-Ferrell-Larkin-Ovchinnikov state in a magnetic-field-induced organic superconductor. Phys. Rev. Lett. 97, $157001(2006)$

14. Vorontsov, A. B., Sauls, J. A. \& Graf, M. J. Phase diagram and spectroscopy of Fulde-Ferrell-Larkin-Ovchinnikov states of two-dimensional $d$-wave superconductors. Phys. Rev. B 72, 184501 (2005).

15. Agosta, C. C. et al. Experimental and semiempirical method to determine the Pauli-limiting field in quasi-two-dimensional superconductors as applied to $\kappa$-(BEDT-TTF $)_{2}(\mathrm{CuNCS})_{2}$ : Strong evidence of a FFLO state. Phys. Rev. B 85, 214514 (2012).

16. Wright, J. A. et al. Zeeman-driven phase transition within the superconducting state of $\kappa$-(BEDT-TTF $)_{2}(\mathrm{CuNCS})_{2}$. Phys. Rev. Lett. 107, 087002 (2011).

17. Kawamoto, A., Miyagawa, K., Nakazawa, Y. \& Kanoda, K. ${ }^{13} \mathrm{C}$ NMR study of layered organic superconductors based on BEDT-TTF molecules. Phys. Rev. Lett. 74, 3455-3458 (1995). 
18. Mayaffre, H., Wzietek, P., Jérome, D., Lenoir, C. \& Batail, P. Superconducting state of $\kappa-(\mathrm{ET})_{2} \mathrm{Cu}\left[\mathrm{N}(\mathrm{CN})_{2}\right] \mathrm{Br}$. Studied by ${ }^{13} \mathrm{C}$ NMR: Evidence for vortex-core-induced nuclear relaxation and unconventional pairing. Phys. Rev. Lett. 75, 4122-4125 (1995)

19. Mayaffre, H., Wzietek, P., Lenoir, C., Jérome, D. \& Batail, P. ${ }^{13} \mathrm{C}$ NMR study of a quasi-two-dimensional organic superconductor $\kappa-(\mathrm{ET})_{2} \mathrm{Cu}\left[\mathrm{N}(\mathrm{CN})_{2}\right] \mathrm{Br}$. Europhys. Lett. 28, 205-210 (1994).

20. De Soto, S. M. et al. ${ }^{13} \mathrm{C}$ NMR studies of the normal and superconducting states of the organic superconductor $\kappa-(\mathrm{ET})_{2} \mathrm{Cu}\left[\mathrm{N}(\mathrm{CN})_{2}\right]$ Br. Phys. Rev. B 52, 10364-10368 (1995).

21. Abragam, A. Principles of Nuclear Magnetism (Oxford Univ. Press, 1999).

22. Clem, J. R. \& Coffey, M. W. Viscous flux motion in a Josephson-coupled layer model of high- $T_{\mathrm{c}}$ superconductors. Phys. Rev. B 42, 6209-6216 (1990).

23. De Soto, S. M., Slichter, C. P., Wang, H. H., Geiser, U. \& Williams, J. M. Evidence for the role of fluxoids in enhancing NMR spin-lattice relaxation and implications for intrinsic pinning of the flux lattice in organic superconductors. Phys. Rev. Lett. 70, 2956-2959 (1993).

24. Mansky, P. A., Chaikin, P. M. \& Haddon, R. C. Vortex lock-in state in a layered superconductor. Phys. Rev. Lett. 70, 1323-1326 (1993).

25. Mansky, P. A., Chaikin, P. M. \& Haddon, R. C. Viscous flux motion in a Josephson-coupled layer model of high- $T_{\mathrm{c}}$ superconductors. Phys. Rev. B 50, 15929-15944 (1994).

26. Won, H. et al. Upper critical field and Fulde-Ferrell-Larkin-Ovchinnikov state

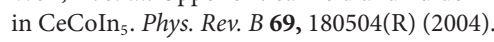

27. Wang, Q., Chen, H-Y., Hu, C-R. \& Ting, C. S. Local tunneling spectroscopy as a signature of the Fulde-Ferrell-Larkin-Ovchinnikov State in $s$ - and $d$-wave superconductors. Phys. Rev. Lett. 96, 117006 (2006).

28. Yanase, Y. \& Sigrist, M. Antiferromagnetic order and $\pi$-triplet pairing in the Fulde-Ferrell-Larkin-Ovchinnikov state. J. Phys. Soc. Jpn 78, 114715 (2009).
29. Cui, Q., Hu, C-R., Wei, J. Y. T. \& Yang, K. Spectroscopic signatures of the Larkin-Ovchinnikov state in the conductance characteristics of a normal-metal/superconductor junction. Phys. Rev. B 85, 014503 (2012).

30. Urayama, H. et al. A new ambient pressure organic superconductor based on BEDT-TTF with $T_{C}$ higher than $10 \mathrm{~K}\left(T_{C}=10.4 \mathrm{~K}\right)$. Chem. Lett. 17, 55-58 (1988).

\section{Acknowledgements}

We would like to thank A. Vorontsov, Y. Yanase and M. Sigrist for illuminating discussions, and I. Sheikin for providing raw data for the phase diagram. This research is supported by funds from the French ANR grant 06-BLAN-0111, the EuroMagNET II network under EU Contract No. 228043, the visiting faculty program of Université Joseph Fourier (V.F.M.), and ADVANCE HRD-0548311 (V.F.M.).

\section{Author contributions}

K.M. and K.K. prepared the samples. H.M., S.K., K.M. and V.F.M. performed the experiments. S.K. and M.H. developed and operated the high-field NMR facility. H.M. created software for the spectrometers. H.M. and V.F.M. analysed the data. C.B. provided conceptual advice and contributed to the planning of the project. H.M., C.B., M.H. and V.F.M. developed the data interpretation. V.F.M. wrote the paper and supervised the project. All authors discussed the results and commented on and edited the manuscript.

\section{Additional information}

Supplementary information is available in the online version of the paper. Reprints and permissions information is available online at www.nature.com/reprints. Correspondence and requests for materials should be addressed to V.F.M.

\section{Competing financial interests}

The authors declare no competing financial interests. 